\title{
Coupling Effects of Dual SiGe Power Amplifiers for 802.11n MIMO Applications
}

\author{
Wei-Chun Hua ${ }^{1}$, Po-Tsung Lin ${ }^{1}$, Chun-Ping $\operatorname{Lin}^{1}$, Che-Yung Lin ${ }^{1}$, Huan-Lin Chang ${ }^{1}$, Chee Wee Liu ${ }^{1,}$, \\ Tzu-Yi Yang ${ }^{2}$, and Gin-Kou $\mathrm{Ma}^{2}$ \\ ${ }^{1}$ Department of Electrical Engineering and Graduate Institute of Electronics Engineering, National \\ Taiwan University, Taiwan, R. O. C. *chee@cc.ee.ntu.edu.tw \\ ${ }^{2}$ SoC Technology Center, Industrial Technology Research Institute, Taiwan, R.O.C.
}

\begin{abstract}
The large-signal and small-signal coupling effects of dual SiGe power amplifiers (PAs) on a single chip for 802.11n Multiple Input Multiple Output (MIMO) applications are demonstrated for the first time. Deep trench isolation and grounded guard ring are used for crosstalk isolation at both transistor and circuit levels. The equivalent small-signal coupling at $2.45 \mathrm{GHz}$ between two PAs is -30 dB. The PA delivers $18.1 \mathrm{dBm}$ and $16.6 \mathrm{dBm}$ with $3 \% \mathrm{EVM}$ (OFDM, 64-QAM) in single and dual PA operation modes, respectively. The EVM degradation becomes severe as the relative interfering power level increases.
\end{abstract}

Index Terms - wireless LAN, MIMO, coupling, crosstalk, isolation, and power amplifiers.

\section{INTRODUCTION}

Due to the rapidly increasing demands on high throughput wireless data transmission, a new IEEE $802.11 \mathrm{n}$ standard targeting on throughput larger than 100 Mbps attracts great attention. To have higher throughput in the same bandwidth, spectrum-efficient modulation, such as OFDM is adopted in the IEEE 802.11n standard. Some minor revisions of the modulation scheme of the OFDM signal used in the $802.11 \mathrm{a} / \mathrm{g}$ standards are made to further increase the spectral efficiency, such as higher coding rate, shorter guard interval, and larger constellation size. The modified OFDM signal results in stricter linearity requirement of the $802.11 \mathrm{n}$ PA than the $802.11 \mathrm{a} / \mathrm{g}$ PA.

Besides the influence caused by the modified modulation scheme, another challenge comes from the MIMO transceiver architecture. The latest 802.11n proposal adopts spatial multiplexing to increase the throughput to 2 and 4 times in $2 \times 2$ and $4 \times 4$ modes, respectively. In point of cost reduction, multiple PAs in the MIMO system should be placed in a single chip, but the crosstalk between PAs under large-signal operation is still a big concern. No systematic study on the large-signal coupling effect of the realistic chip is found in the literatures, only simulation work at system level [1] is proposed so far.

In order to clarify the coupling effect on the linearity performance of the PA, dual SiGe PAs with symmetric layouts on a single chip is fabricated and the systematic study of the coupling effects between two PAs are reported in this work.

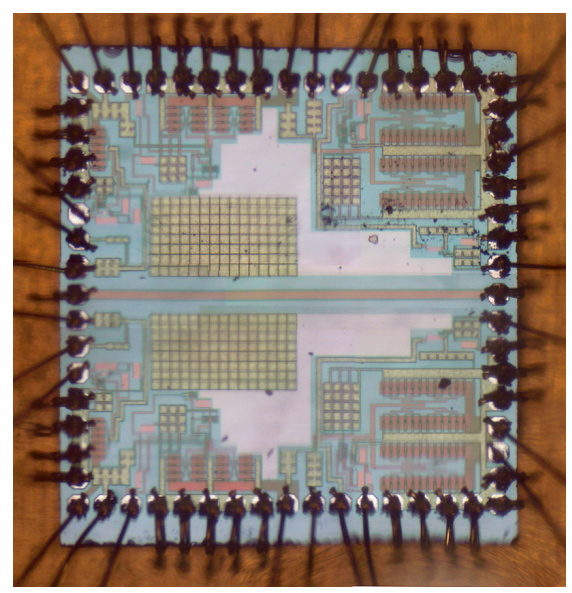

Fig. 1 Chip (1.68 mm x $1.68 \mathrm{~mm})$ photos of the $802.11 \mathrm{n}$ MIMO PA.

\section{DESIGN OF THE 802.11N POWER AMPLIFIER}

Fig. 1 shows the chip $(1.68 \mathrm{~mm} \times 1.68 \mathrm{~mm})$ photo of the PA. Each unit transistor of the PA is surrounded by the grounded guard ring [2] and deep trench [2], [3] to minimize the coupling effect. The grounded guard ring with deep trenches at both sides is also placed in the middle of the PA chip for crosstalk isolation. Although many other effective isolation techniques are proposed in the literatures, such as porous $\mathrm{Si}$ trench [4], silicon-oninsulator (SOI) substrate [2], and high resistivity substrate obtained by the proton bombardment [5], all of them need special treatment that are not included in the standard BiCMOS process. Thus the combination of the deep trench isolation and the grounded guard ring is still the most attractive choice for the designers with the standard BiCMOS process. 


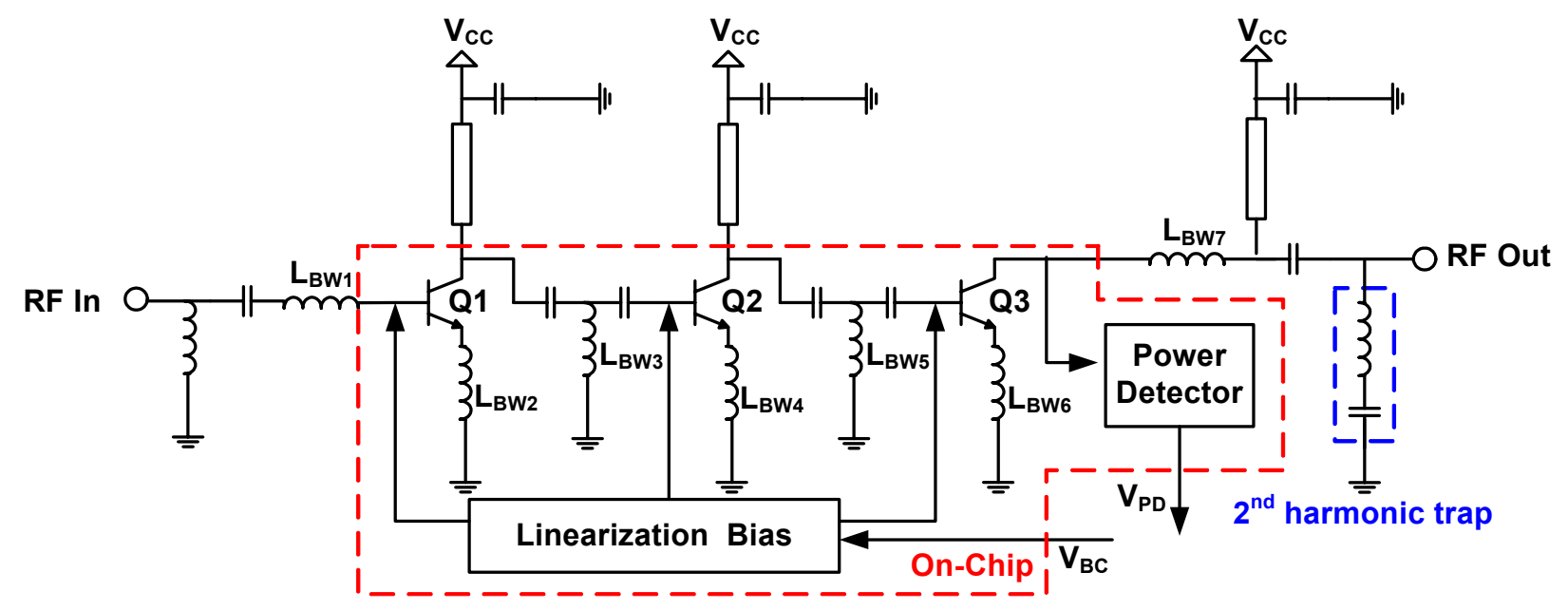

Fig. 2 Simplified circuit diagram of the power amplifier.

Fig. 2 shows the simplified circuit diagram of the single PA for the 802.11n applications. The PA consists of three single-ended common-emitter stages with integrated active bias linearization and power detector (PD). The PA is also optimized to be temperature-insensitive. The operating principle and design methodology of the bias circuit and the PD can be found in our previous work [6]. With the proper designed high-pass inter-stage matching network, the PA doesn't have any on-chip low-Q inductor and is an inductor-less chip. All the inductors of the PA are achieved by the high-Q bond-wire inductors and surface mounted inductors. The bias current of each stage can be controlled by the bias control voltage $\left(\mathrm{V}_{\mathrm{BC}}\right)$, thus the bias current can be reduced at low $\mathrm{P}_{\text {out }}$ level to improve the power-added-efficiency (PAE). The $2^{\text {nd }}$ harmonic trap is added in the output matching network to sink the $2^{\text {nd }}$ harmonic signal to ground and improves the linearity of the PA with the $2^{\text {nd }}$ harmonic rejection of -60 $\mathrm{dBc}$.

\section{EXPERIMENTAL RESULTS}

Fig. 3 shows the power characteristics of one PA at $2.45 \mathrm{GHz}$ on the dual PA chip. The DC supply voltage and the quiescent current of the PA are $3.3 \mathrm{~V}$ and $124 \mathrm{~mA}$, respectively. The small signal gain, $\mathrm{P}_{1 \mathrm{~dB}}$, and $\mathrm{P}_{\text {sat }}$ are 26.7 $\mathrm{dB}, 24.3 \mathrm{dBm}$, and $25.2 \mathrm{dBm}$, respectively. The gain expansion is $1.4 \mathrm{~dB}$ which results from the linearization bias. The PAE at $802.11 \mathrm{~b} / \mathrm{g}$ linear $\mathrm{P}_{\text {out }}(22 / 18.1 \mathrm{dBm})$ are $11.8 \%$ and $7.8 \%$, respectively. Although the total emitter area of the final stage for a single PA $\left(1754 \mu \mathrm{m}^{2}\right)$ is shrunk to the half of the previous work $\left(3508 \mu \mathrm{m}^{2}\right)$, the reduction of $\mathrm{P}_{\text {out }}$ is less than $3 \mathrm{~dB}$ due to the slightly increased bias current density and the addition of the $2^{\text {nd }}$ harmonic trap.

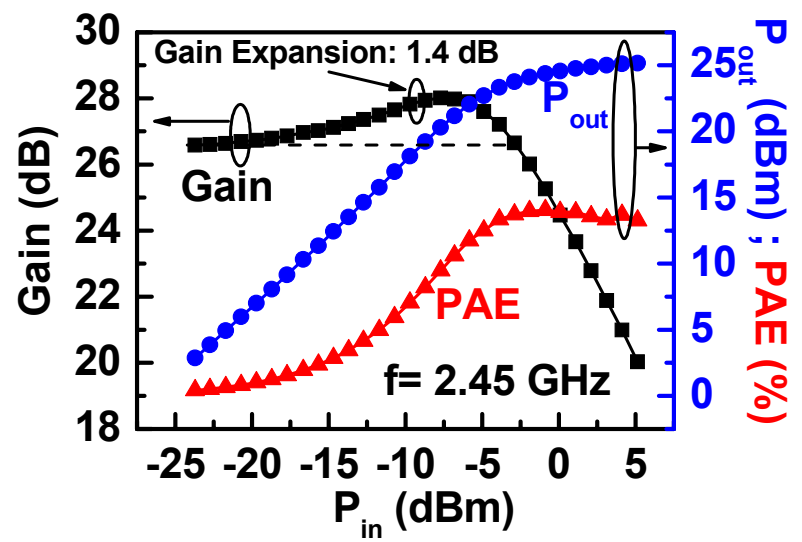

Fig. 3 The power characteristics of one PA at $2.45 \mathrm{GHz}$ on the dual PA chip.

\section{A. Small- Signal Coupling Effect}

Fig. 4 shows the illustration of the PCB and the assumed signal flow under 4-port small signal measurement. The $S 41$ is not able to stand for the coupling effects since it also includes the small signal gain of the PA. The more reasonable expression of the equivalent on-chip coupling effect is $S 4^{\prime} 1^{\prime}-S 2^{\prime} 1^{\prime}$ in $\mathrm{dB}$ when both PAs turn on, which excludes the small signal gain of one PA. The equivalent coupling stands for the total amount of the crosstalk between up-PA and downPA along the entire signal path on the chip. Although the vertical coupling occurs at any location of the signal path of the PA, we assume that any signal path from port- 1 to port-4 is equivalent to the combination of the complete amplification of a single PA and the vertical coupling between PAs. The coupling effect on PCB is neglected 


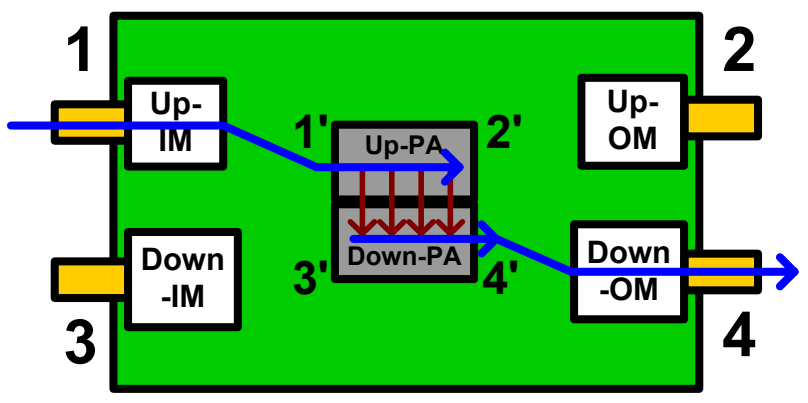

Fig. 4 The illustration of the PCB and signal flow under 4 -port small signal measurement.

since the distance between up path and down path on the PCB is much larger than that of the on-chip case. Moreover, since $S 4^{\prime} 1^{\prime}$ and $S 2^{\prime}{ }^{\prime}$ ' can not be directly measured through the ports of the PCB, an approximated expression of the on-chip equivalent coupling is given in (1). The units of the S-parameters in (1) are all dB. Note that $\left|S 44^{\prime}\right|$ and $\left|S 22^{\prime}\right|$ are approximately equal when both PAs turn on due to the symmetry of up-PA and down-PA. Thus, $\left|S 4^{\prime} 1^{\prime}\right|-\left|S 2^{\prime} 1^{\prime}\right|(\mathrm{dB})$ can represent the magnitude of the equivalent on-chip coupling and is approximated to $|S 41|$ - |S21| (dB) by (1).

$$
\begin{gathered}
\left|S 4^{\prime} 1^{\prime}\right|-\left|S 2^{\prime} 1^{\prime}\right|=\left(|S 41|-\left|S 1^{\prime} 1\right|-\left|S 44^{\prime}\right|\right) \\
-\left(|S 21|-\left|S 1^{\prime} 1\right|-\left|S 22^{\prime}\right|\right) \cong|S 41|-|S 21|(d B)
\end{gathered}
$$

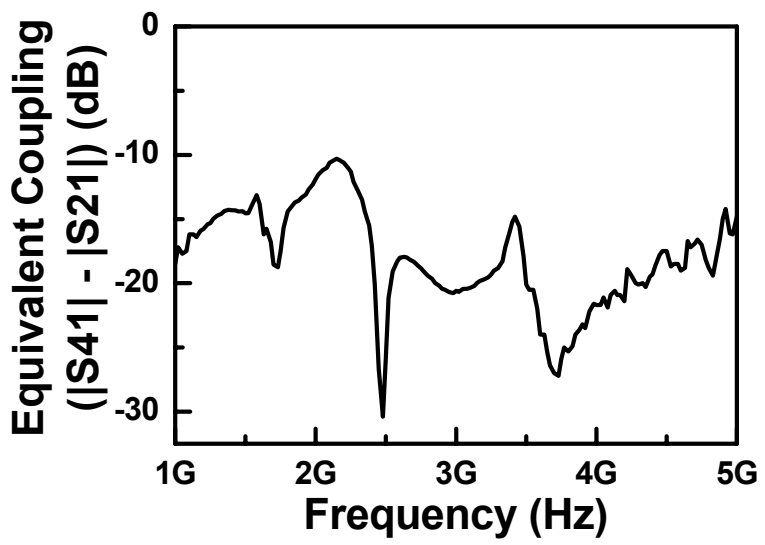

Fig. 5 The equivalent on-chip coupling between two PAs.

Fig. 5 shows the equivalent small-signal on-chip coupling between two PAs. The coupling is lowest (-30 $\mathrm{dB})$ at the operating frequency $(2.45 \mathrm{GHz})$ which means that the signal prefers the horizontal signal path which exhibits the matched impedance rather than the coupling path with unmatched impedance at the operating frequency. To design the PA with well matched impedance will inherently reject the on-chip crosstalk at the same time.
B. Coupling Effect on S-parameters with $2.45 \mathrm{GHz}$ OFDM Modulation Signal of Different Power Levels

Fig. 6 shows the S-parameters of the up-PA when the down-PA operates with $2.45 \mathrm{GHz}$ OFDM modulation signal at two power levels: (1) The same $\mathrm{P}_{\text {in }}$ as the up-PA $(-25 \mathrm{dBm})(2) 25 \mathrm{~dB}$ larger than the up-PA $(0 \mathrm{dBm})$. When the down-PA operates at the same $\mathrm{P}_{\text {in }}$ as the up-PA, the S-parameters of the up-PA are almost the same as the up-PA operates alone ((a) and (c) in Fig. 6). When the $P_{\text {in }}$ of the down-PA is larger than the up-PA by $25 \mathrm{~dB}$, the amount of the coupling signal from down-PA is of the same order of the signal propagated in the up-PA. Thus, significant coupling occurs and which results in spikes of the S-parameters of the up-PA ((b) and (d) in Fig. 6).

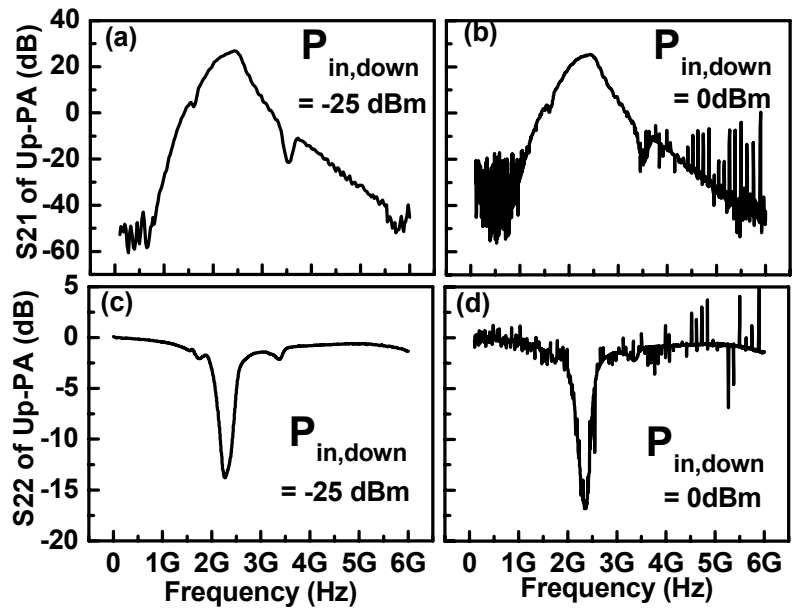

Fig. 6 The S-parameters of the up-PA when the down-PA operates with $2.45 \mathrm{GHz}$ OFDM modulation signal at two power level: (a) and (c) The same $P_{\text {in }}$ as the up-PA (-25 $\mathrm{dBm})$; (b) and (d) $25 \mathrm{~dB}$ larger than the up-PA (0 dBm).

\section{Large-Signal Coupling Effect on EVM}

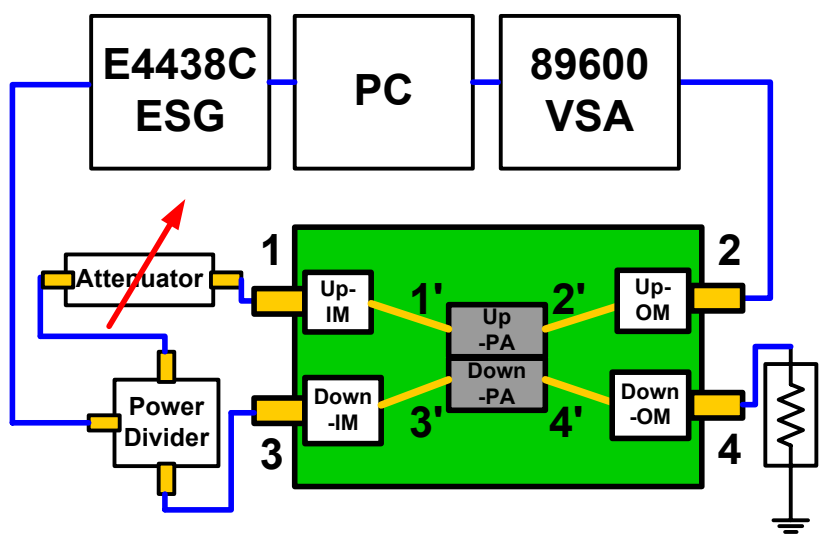

Fig. 7 The measurement setup of the coupling effect on EVM under large signal operation. 
Fig. 7 shows the measurement setup of the large signal coupling effect on EVM. The power divider is used at the source end to obtain two modulation signals (OFDM, 64QAM) from one source. The relative input power levels between up-PA and down-PA can be adjusted by the attenuator.

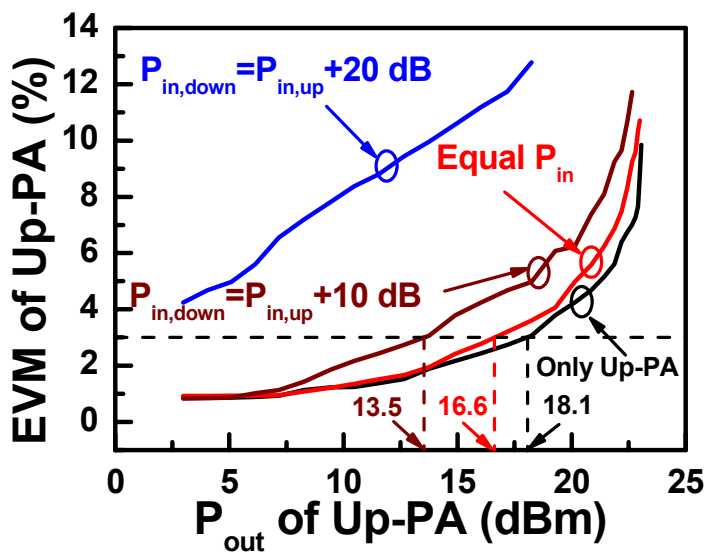

Fig. 8 The EVM of the up-PA with the interference from the down-PA with different input power levels.

Fig. 8 shows the EVM of the up-PA with the interference from the down-PA with various input power levels with respect to the up-PA. Since the $802.11 \mathrm{n}$ standard is not available so far, the modulation signal of 802.11a/g (OFDM, 64QAM) is used in the EVM measurements. The linear $\mathrm{P}_{\text {out }}$ of the up-PA is $18.1 \mathrm{dBm}$ at EVM of 3\% for single PA operation mode and degrades $1.5 \mathrm{dBm}$ when down-PA turns on with the same $\mathrm{P}_{\text {in }}$ as the up-PA. The linearity is further degraded if the $\mathrm{P}_{\text {in }}$ of the down-PA is larger than the up-PA by $10 \mathrm{~dB}$ and $20 \mathrm{~dB}$. The performance summary of the PA is listed in Table I and the performance under single PA operation mode is comparable to the $802.11 \mathrm{~b} / \mathrm{g}$ SiGe PA products.

\section{CONCLUSION}

Although the MIMO transceiver can dramatically increase the throughput of the wireless data transmission by several times, the on-chip crosstalk between multiple PAs is not negligible. In this work, even the deep trench isolation and grounded guard ring can reject the equivalent on-chip coupling up to $-30 \mathrm{~dB}$ at $2.45 \mathrm{GHz}$, the linear $\mathrm{P}_{\text {out }}$ still degrades $1.5 \mathrm{dBm}$ under dual PA operation mode with the same $\mathrm{P}_{\text {in }}$. More effective isolation technology is needed to lower the on-chip coupling and maintain the linearity of multiple PAs on a single chip in order to achieve the single-chip MIMO transceiver.
TABLE I

PERFORMANCE SUMMARY

\begin{tabular}{|c|c|}
\hline Frequency $(\mathrm{GHz})$ & 2.45 \\
\hline $\begin{array}{c}\text { DC Bias Voltage }(\mathrm{V}) \text { and } \\
\text { Current }(\mathrm{mA})\end{array}$ & $3.3 ; 124$ \\
\hline Linear Gain $(\mathrm{dB})$ & 26.7 \\
\hline $\begin{array}{c}\text { Gain Flatness }(\mathrm{dB}) \\
(2.4 \sim 2.5 \mathrm{GHz})\end{array}$ & 0.4 \\
\hline $2^{\text {nd }}$ harmonic rejection $(\mathrm{dBc})$ & -60 \\
\hline $\mathrm{P}_{\text {sat }}(\mathrm{dBm})$ & 25.2 \\
\hline $\mathrm{P}_{1 \mathrm{~dB}}(\mathrm{dBm})$ & 24.3 \\
\hline Linear $\mathrm{P}_{\text {out }}(\mathrm{dBm})$ & $\begin{array}{c}22.0(11 \mathrm{~b}) ; 18.1(11 \mathrm{~g}) \\
16.6(11 \mathrm{~g}, \mathrm{dual} \mathrm{PA})\end{array}$ \\
\hline PAE @ linear $\mathrm{P}_{\text {out }}(\%)$ & $11.8(11 \mathrm{~b}) ; 7.1(11 \mathrm{~g})$ \\
\hline $\begin{array}{c}\text { Equivalent Coupling } \\
\text { @ 2.45 GHz }(\mathrm{dB})\end{array}$ & -30 \\
\hline Power Detector Voltage $(\mathrm{V})$ & $0.7-1.7(5-25 \mathrm{dBm})$ \\
\hline
\end{tabular}

\section{ACKNOWLEDGMENT}

This work is supported by the Winbond Electronics Corporation under contract no. 93-S-B50. The authors would also like to acknowledge the fabrication support provided by National Chip Implementation Center (CIC).

\section{REFERENCES}

[1] S. Woo, D. Lee, K. Kim, Y. Hur, C.-H. Lee, J. Laskar, "Combined Effects of RF Impairments in the Future IEEE 802.11n WLAN Systems," IEEE Vehicular Technology Conference, vol. 2, pp. 1346-1349, May 2005.

[2] M. Kumar, Y. Tan, J. K. O. Sin, "Excellent Cross-Talk Isolation, High-Q Inductors, and Reduced Self-Heating in a TFSOI Technology for System-on-a-Chip Applications," IEEE Trans. Electron Devices, vol. 49, no.4, pp. 584-589, April 2002.

[3] C. S. Kim, P. Park, J.-W. Park, N. Hwang, and H. K. Yu, "Deep Trench Guard Technology to Suppress Coupling between Inductors in Silicon RF ICs," IEEE MTT-S Symp. Dig., pp. 1873-1876, June 2001.

[4] H.-S. Kim, K. A. Jenkins, and Y.-H. Xie, "Effective Crosstalk Isolation Through $\mathrm{p}^{+} \mathrm{Si}$ Substrate With SemiInsulating Porous Si,” IEEE Electron Device Lett., vol. 23, no. 3, pp. 160-162, March 2002.

[5] Y. H. Wu, A. Chin, K. H. Shih, C. C. Wu, C. P. Liao, S. C. Pai, and C. C. Chi, "Fabrication of Very High Resistivity Si with Low Loss and Cross Talk," IEEE Electron Device Lett., vol. 21, no. 9, pp. 442-444, September 2000.

[6] W.-C. Hua, H.-H. Lai, P.-T. Lin, C. W. Liu, T.-Y. Yang, and G.-K. Ma, "High-Linearity and TemperatureInsensitive $2.4 \mathrm{GHz}$ SiGe Power Amplifier with DynamicBias Control," IEEE RFIC Symp. Dig., pp. 609-612, June 2005. 